\title{
Prevalence of Depression in Indian Adolescents
}

\author{
Rajesh Sagar $^{1} \cdot$ Nivedhitha Selvakumar ${ }^{1}$
}

Received: 2 March 2021 / Accepted: 19 March 2021/Published online: 6 April 2021

(C) Dr. K C Chaudhuri Foundation 2021

Depressive disorders are among the most common mental health problems affecting around 45.7 million people in India and a major contributor to DALYs (disability-adjusted life-years) [1]. It has been observed that there is a rise in depressive disorders during the postpubertal phase corresponding to the adolescent age group which can be accounted for by various biopsychosocial risk factors. However, the disorder is frequently underdiagnosed in this population and the clinicians are posed with challenges because of its unique presentation with prominent irritability and varying psychopathology [2].

With the rising prevalence of the disorder in this era of internet, social media, and changing milieu, a study on the prevalence of depression is essential to identify and address the unmet needs in the adolescent population [3]. The study has been able to highlight the need for a more careful approach while diagnosing the disorder given its wide prevalence and underdiagnosis. Also, identification of predictors of depression such as family dispute helps in a more cautious approach while encountering children with such risk factors and can aid in preventive management as well.

Center for Epidemiological Studies-Depression Revised (CESD-R) Scale was used to assess for the presence of depression. However, a subsequent detailed clinical interview and examination of the subjects at risk would have given a clear picture to confirm the occurrence of depressive disorders. As it is a self-rated measure, there could have been bias in reporting of information. Hence, taking it as sole measure to diagnose depressive disorder may have its limitations.

The study found that $14.19 \%$ of the adolescents had depression. A score of 16 or above on CESD-R indicated that a person is at risk for depression. This includes subthreshold depressive symptoms as well. It is essential to capture this

Rajesh Sagar

rsagar29@gmail.com

1 Department of Psychiatry, All India Institute of Medical Sciences, Ansari Nagar, New Delhi 110029, India population as well, because such adolescents may be at risk for developing a syndromal episodes in future. However, the prevalence of syndromal depression requiring active intervention might have been lesser than observed in the study, when the subsyndromal cases are not considered.

Suicide is among the leading cause of death in this population [4]. The CESD-R has an item for suicidal ideation. Children who are scoring high on this might require immediate clinical attention. A more detailed assessment and interpretation of this item would increase the clinical significance of the study; $9.08 \%$ reported learning disability. The distinction has to be drawn between children having learning disability and those with only learning difficulty. As this was reported by the subjects themselves and was not assessed with validated tools, one has to be cautious while interpreting the results. Children with learning disability are more prone to develop depressive disorder because of the psychosocial risk factors [5].

The study had estimated the prevalence of depression in school-going adolescents. However, one has to be cautious while extrapolating this finding to the adolescents in the general community. There is a possibility of exclusion of adolescents who have never enrolled or have dropped out of school. Such adolescents have a greater risk of developing a depressive episode and this could be a limitation in the school-based study.

Hence, future studies focussing on a wider population and detailed evaluation in high-risk groups can add valuable evidence to formulate management guidelines and policies in adolescents.

\section{Declarations}

Conflict of Interest None.

\section{References}

1. Sagar R, Dandona R, Gururaj G, et al. The burden of mental disorders across the states of India: the global burden of disease study 1990-2017. Lancet Psychiatry. 2020;7:148-61. 
2. Sagar R, Pattanayak RD, Mehta M. Clinical profile of mood disorders in children. Indian Pediatr. 2012;49:21-3.

3. Paul B, Usha VK. Prevalence and predictors of depression among adolescents. Indian J Pediatr. 2020. https://doi.org/10.1007/s12098020-03491-w.

4. Windfuhr K, While D, Hunt I, et al. National confidential inquiry into suicide and homicide by people with mental illness. Suicide in juveniles and adolescents in the United Kingdom. J Child Psychol Psychiatry. 2008;49:1155-65.
5. Wright-Strawderman C, Watson BL. The prevalence of depressive symptoms in children with learning disabilities. J Learn Disabil. 1992;25:258-64.

Publisher's Note Springer Nature remains neutral with regard to jurisdictional claims in published maps and institutional affiliations. 